adelantar algunas conclusiones. El periodismo africano, cristiano y católico, no es ni buen periodismo, ni genuinamente cristiano y católico. Lo que se necesita, por lo tanto, es: a) una mejor formación y mayor profesionalismo; b) una concepción cristiana más profunda de la prensa; c) una visión más "católica"; d) tomar más en serio a Africa y a sus problemas; e) una información que aprofundice y una política editorial responsable, nacida del sentimiento de responsabilidad social; f) crear un ambiente de comprensión, favorable a una prensa libre, $\mathrm{y}$, finalmente $\mathrm{g}$ ) emprender una séria investigación sobre las Comunicaciones. A pesar de sus numerosas deficiencias, existen motivos de esperanza para la Prensa Africana Cristiana.

\title{
Die Centrums-Parlaments-Correspondenz (CPC)
}

\author{
von Michael Bornefeld-Ettmann
}

\section{Der Augustinusverein als Initiator}

Mit der Verschärfung des Kulturkampfes wuchs das Interesse der Deutschen Zentrumspartei an einem guten politischen und organisatorischen Verhältnis zur katholischen Presse, die bis dahin nicht als parteigebunden im engeren politischen Sinne anzusehen war.

Auf einer der Versammlungen katholischer Organisationen sahen sich denn auch Vertreter der Zentrumspartei, der Kirche und der Presse veranlaßt, Schritte zur Verbesserung des Verhältnisses zwischen Presse und Partei zu unternehmen. ${ }^{1}$ Wie so oft bei derartigen Unterfangen konzentrierten sich auch hier die Bemühungen zunächst auf die Errichtung einer Informations-Institution zwischen der bestehenden Organisation (Zentrumspartei) und dem noch vereinzelten Partner (Presse).

So wurde hier, während der Tagung des "Mainzer Katholikenvereins" (15. bis 17. 6. 1874), auf Anregung des Düsseldorfer Pfarrers M. Prell, des Redakteurs beim „Düsseldorfer Volksblatt", Dr. E. Hüsgen, und des MdR Dr. E. Lieber der Plan zur Gründung eines Zentralbüros für die katholische Presse gefaßt. Aufgabe dieses Zentralbüros sollte die Herausgabe einer Parlamentskorrespondenz sein, die aus Berlin von den Verhandlungen des Reichstages und des Preußischen Landtages an die Presse berichten sollte.

Mit den Vorbereitungen dazu wurde der Redakteur der "Germania“, Friedrich Nienkemper, beauftragt, der sich in einem Rundschreiben vom 18. 8. 1874 an alle katholischen Redaktionen und Verleger wandte. Darin legt er sein Programm für die Gründung einer „Parlaments-Correspondenz" (PC) dar und schlägt die Einberufung eines Journalisten- und Verlegertages vor, welcher über einen generellen Zusammenschluß der katholischen Presse beraten soll.

Damit ist festzustellen, daß die Vorgeschichte der Centrums-Parlaments-Correspondenz (CPC) und die der agilsten und beständigsten Vereinigung katholischer Presse in Deutschland - des "Augustinusvereins zur Pflege der katholischen Presse

Michael Bornefeld-Ettmann studiert (Doktorand) an der Universität Münster Publizistik, Germanistik und Soziologie. Er ist Mitarbeiter studentischer Zeitschriften. 
Deutschlands $s^{*}$ - parallel laufen, ja, daß sogar die Gründung eines Pressevereins im Aufbau einer parlamentarischen Korrespondenz einen ihrer Anlässe hatte.

Die am Ziel der Kooperation zwischen Presse und Partei orientierte Planung ruft den Zusammenschluß der Presse hervor; ein Schritt zur Angleichung der Organisationssysteme, - bedeutsame Erscheinung im Vorfeld publizistischer Zusammenhänge.

Der von Nienkemper geplante Zusammenschluß kam nicht in erhofftem Umfang zustande, so daß die PC nur vom Oktober 1874 bis September 1875 erscheinen konnte. Von der "Germania"-Redaktion unter Leitung Nienkempers erstellt, standen ihr nicht genug Mittel zur Verfügung, so daß die Verlagsdirektion die weitere Herausgabe untersagen mußte. Damit zerfielen auch die übrigen Initiativen des ,Vereins von Verlegern katholischer Zeitungen".

Ein Versuch des Trierer Kaplans F. Dasbach, einen Verband katholischer Verleger und Redakteure zur Fortführung des PC-Büros ins Leben zu rufen, schlug im Oktober 1875 fehl.2

Für drei Monate übernahm ein von der „Schlesischen Volkszeitung “ (Breslau) gegründetes Neues Parlaments-Correspondenzbüro (NPC) die Funktion der PC, dann „... zu Beginn des Jahres 1876 war die katholische Presse wieder ohne jedes äußere Band, da mit dem Eingehen der Korrespondenz auch der Verleger-Verein wieder auseinanderfiel. ${ }^{\text {" }}$

Der Gedanke an einen Zusammenschluß der katholischen Presse und an die Gründung einer parlamentarischen Korrespondenz als informatorisches Bindeglied zwischen (Zentrums)-Presse und -Partei war jedoch nun einmal aufgetaucht und hatte die ersten konkreten Erfolge gezeitigt. Er gewann an Boden und wurde weiter präzisiert. So formulierten der Düsseldorfer Kaplan Dr. H. J. Schmitz und der Redakteur Dr. E. Hüsgen auf der Würzburger Katholikenversammlung von 1877 die früheren Anregungen neu und gaben damit den wohl entscheidenden Anstoß. So „... kam es... zu einer guten Organisation der katholischen und ZentrumsPresse. Sie gab sich den Namen ,Augustinusverein'. Die Gründung geschah am 15. 5. 1878 in Düsseldorf, endgültig am 27. 8. $1878 \mathrm{zu} \mathrm{Köln.} \mathrm{“4}$

War die Erstellung einer Informations-Instanz zwischen Presse und Partei einer der hauptsächlichen Anlässe zur Gründung des Pressevereins gewesen, so lag auch das erste Interesse dieses neuen Verbandes darin, jene publizistische Institution zu schaffen. So besagt der $₫ 1$ der Statuten des Augustinusvereins: „Der Augustinusverein hat den Zweck, die katholische Tagespresse in Deutschland dadurch zu fördern, daß er ... für die bestehenden Tagesblätter eine gemeinsame quellenmäßige Information und Berichterstattung herstellt. ${ }^{\alpha_{5}}$

Diese Bestimmung wurde von vornherein als politische Aufgabe begriffen, lenkte den Verein während seiner gesamten Geschichte und machte ihn somit selbst $z u$ einer verbindenden, in beide Richtungen wirkenden Institution zwischen Partei und Presse. Damit war der Rahmen einer ausschließlichen Presse-Organisation bereits vom Ansatz her überschritten und ging in Richtung einer bewußt politischen Mediatisierung. Dazu der Zentrums-Historiker Bachem: "Ursprünglich gedacht als Standesverein zur Förderung... der gesamten Presse der Katholiken, sowohl der politischen als auch der katholisch-religiösen, konnte er sich... der Aufgabe nicht entziehen, auf eine möglichst einheitliche politische Haltung der $\mathrm{zu} \mathrm{ihm}$ gehörenden politischen Blätter Bedacht zu nehmen. ${ }^{{ }^{\circ}}$

Obwohl die politische Einflußnahme nur in Richtung der Zentrumspartei gehen konnte, ergibt sich für den Beobachter der Eindruck, als habe sich der Augustinus- 
verein und mit $\mathrm{ihm}$ sein Zögling CPC mit allen Mitteln um die politische und publizistische Anerkennung durch die Zentrumspartei bemüht, sei aber dort weitgehend auf Unverständnis gestoßen, so daß der Vermittlungs- und Verbindungsauftrag zwischen Partei und Presse sich lange Zeit nur auf die Presse hin artikulieren konnte. ${ }^{7}$

Noch so manches Mal klagte der Augustinusverein über das geringe Entgegenkommen, das seitens der Zentrumspartei und ihrer Fraktionen Verein und Presse gegenüber bewiesen worden sei. Auf der anderen Seite klingen ebensolche Vorwürfe der Partei an: Angriffe gegen das Selbständigkeitsstreben des Augustinusvereins und der Presse sind Legion. Während man in der Rede des Vorsitzenden des Augustinusvereins, H. Otto, auf dem Katholikentag 1903 in Köln - „In seiner politischen Tätigkeit hat er [der Augustinusverein; Anm. d. Verf.] sich vor dem großen Fehler gehütet, selbständig Zentrumspolitik... machen zu wollen. Er hat sich als Werkzeug betrachtet, Zentrumspolitik zu unterstützen und zu vermitteln ${ }^{\text {“g }}$ apologetische Intentionen vermuten kann, erklären neuere Untersuchungen die Ursache der ,traditionell gespannten Beziehungen zwischen der Zentrumspartei und der Zentrums-Presse " mit dem ausgesprochen starken Unabhängigkeitsstreben der Presse.10 In jedem Falle scheint erwiesen, - und das spielt für die Gründung, den Umraum und die Geschichte der CPC eine erhebliche Rolle -, daß es mit dem Verhältnis Zentrumspresse-Zentrumspartei während des gesamten hier behandelten Zeitraums nicht zum Besten stand.

Trotz der hier wiedergegebenen Diskussionen um die Stellung der Presse und ihres Verbandes konsolidiert sich dieser rasch und breitet sich aus zu einer der größten und wirkungsvollen Presseorganisationen in der deutschen Geschichte. Eine seiner beachtlichen Leistungen war die Etablierung der CPC, jenes Informationskanals zwischen Zentrums-Presse und -Partei.

\section{Die Centrums-Parlaments-Correspondenz (CPC)}

„Für die katholische Presse war es namentlich in der Kulturkampfzeit ein dringendes Bedürfnis, von den großen, meist sehr antikatholisch eingestellten Telegraphenbüros unabhängig zu sein..."11

So ging denn der Augustinusverein bereits in seiner 1. Generalversammlung (5./6. 1. 1879, Köln) das Problem der parlamentarischen Nachrichtenübermittlung an. Kaplan Dasbach (Trier), der Erste Vorsitzende des Augustinusvereins (AV), entwickelte einen ausführlichen Plan zur Gründung einer "Parlamentarischen Correspondenz". Diese sollte vom Parlaments-Redakteur der "Germania" verfaßt und von den abonnierenden Blättern getragen werden. Eine Entscheidung darüber konnte erst in der 2. Generalversammlung des AV (29./30. 3. 1879, Düsseldorf) herbeigeführt werden. Sie vollzog die Gründung der „Centrums-ParlamentsCorrespondenz" und klärte weiter die Finanzierung, die Organisation des CPCBüros und die inhaltliche Struktur der CPC.

\section{Konflikte erschweren die Organisation}

Schon beim zweiten Schritt zun Aufbau der CPC ergaben sich Hindernisse in Fülle: im AV wurden Bedenken dagegen laut, den Ersten Vorsitzenden des AV, Dasbach, zur Errichtung des CPC-Büros nach Berlin zu entsenden, da man auf 
Grund seines geistlichen Standes Schwierigkeiten befürchtete. Dieses „Kulturkampfzeitbild"12 macht den Hintergrund deutlich, vor dem damals ein katholischer Verein operieren mußte. Mit Dr. E. Hüsgen als Mitarbeiter richtete Dasbach das Büro in der Reichshauptstadt ein und gewann den „Germania“-Redakteur F. Nienkemper zum ständigen Leiter der neuen Korrespondenz.

Administrativ wurde die CPC von einer Kommission des AV besorgt. Eine langdauernde scharfe Diskussion um die Eigentumsrechte an der CPC, - die Verwaltung sollte beim AV, die Eigentumsrechte bei den beteiligten Verlagen liegen, macht deutlich, daß die natürlichen Konflikte innerhalb der Verleger-Vereinigung hier auf dem Rücken des Projektes CPC ausgetragen werden und sich verzögernd auswirken.

Nach dreijährigen Auseinandersetzungen legte eine Reihe der Verleger den Entwurf eines Statuts für die CPC vor, das eine finanzielle und organisatorische Trennung vom $A V$ und Umwandlung in eine $\mathrm{GmbH}$ vorsah. Dem Vorsitzenden des AV blieb ein Platz im Aufsichtsrat dieser GmbH vorbehalten. Der bezeichnete Entwurf wurde von der Generalversammlung des AV am 26. 3. 1882 angenommen und die CPC endgültig am 24. 8. 1889 als $\mathrm{GmbH}$ konstituiert.

\section{Finanzieller Engpaß}

Bereits bei Gründung der CPC war über die Finanzierung des Projektes in dem Sinne verhandelt worden, daß die Kosten der Herausgabe M. 900,- pro Monat nicht überschreiten sollten und die Abonnements der vertretenen Verlage diese Kosten würden decken müssen. Die größeren der im AV zusammengeschlossenen Verlage zeichneten sofort monatliche Abonnements-Beiträge, deren Höhe sich nach der Auflage des jeweiligen Blattes richtete. In der Gründungsversammlung erklärten sich folgende Zeitungsverlage zur Mitgliedschaft bereit und zeichneten monatliche Beiträge zwischen M. 110,- und M. 155,-:

1) Kölnische Volkszeitung, Köln; 2) Deutsche Reichszeitung, Bonn; 3) Westfälischer Merkur, Münster; 4) Echo der Gegenwart, Aachen; 5) Essener Volkszeitung, Essen; 6) Niederrheinische Volkszeitung, Krefeld; 7) Trierische Landeszeitung, Trier. Gleichzeitig verpflichteten sich die Mitglieder, über den Voranschlag von M. 900,hinausgehende Kosten gemeinsam zu decken, aufgeschlüsselt wiederum nach der Verlagsgröße.

Die hier dargestellte rechtlich-organisatorische Struktur der CPC konnte - vor allem im finanziellen Bereich - zu Unklarheiten und Reibungen im AV führen.

Das geschah schon mit der ersten Rechnungslegung 1880 und setzte sich in den folgenden Jahren fort. So ergab sich bald, daß die Kosten nicht durch die Abonnements gedeckt werden konnten, sondern immer höhere Zuschüsse seitens der Mitglieder erforderten. Diese nahmen die außerordentlichen Beanspruchungen „... lediglich im Interesse der Zentrumspartei und der Zentrums-Presse auf sich".13

Obwohl im Laufe der Jahre weitere Verlage in die CPC-GmbH eintraten "Germania“, Berlin; "Tremonia“, Dortmund; „Neuß-Grevenbroicher Zeitung“, Neuß; ,Westfälisches Volksblatt", Paderborn - und sich generell Zahl und Auflage der Zentrums-Zeitungen steigerten, verschlechterte sich die finanzielle Lage der CPC bis 1914 immer mehr, da es nicht gelang, die steigenden Kosten des Unternehmens durch eine wesentliche Erhöhung der Abonnentenzahl aufzufangen. ${ }^{14}$ Dieser Zustand lag nicht zuletzt darin begründet, daß die CPC als unabhängige Institution von der Zentrums-Partei nicht offiziell anerkannt war. Über das 
gewöhnlich ohnehin nicht ungetrübte Verhältnis zwischen der Zentrums-Partei und ihren Presseinstitutionen hinaus war die CPC in ihrer spezifischen Mittlerposition häufig in Querelen mit den Parteifraktionen verwickelt und daher in einer nicht sonderlich attraktiven Position für ihre Abonnenten und die übrigen ZentrumsZeitungen. Nach weiterem Rückgang der Abonnentenzahl in den Jahren 1890 bis 1912 sahen sich die wenigen verbliebenen Gesellschafter gezwungen, die Leitung der Zentrums-Partei um eine finanzielle Beteiligung anzugehen.

CPC und AV schlugen in einer Eingabe an den Vorstand der Zentrums-Partei vom 20. 8. 1917 die Schaffung eines $Z$ wangssyndikates vor. Darin sollten von der Partei nur solche Zeitungen als "Zentrums-Zeitungen“ anerkannt werden, die sich an der CPC beteiligen. ${ }^{15}$ Die mißliche Situation der Kriegsjahre ließ jedoch eine Neuregelung nicht gedeihen.

\section{Programm der CPC}

In der Gründungsversammlung 1879 wurden auch Struktur und Form des Inhalts der CPC in einem Programm niedergelegt. Das Programm sah folgende Aufteilung der Berichterstattung vor:

1) Großer Parlamentsbericht mit Stenogramm

2) Kleiner Parlamentsbericht ohne Stenogramm

3) Kleiner Bericht als Resumé

4) Parlamentarische Nachrichten und Informationen aus der Partei.

Aus diesem Programm konnten die abonnierenden Blätter einen oder mehrere Teile beziehen, wobei der "Große Bericht" M. 100,-, das Stenogramm gesondert M. 50,- und der „Kleine Bericht" M. 60,- kosteten.

Das bezeichnete Material erschien vom Jahresende 1879 an „sitzungstäglich“, d. h. an den Tagen, an denen Reichstags- oder Landtagssitzungen stattfanden, durchschnittlich 4- bis 5-mal wöchentlich.16

In den ersten Jahren fast ausschließlich von F. Nienkemper erstellt, lieferte die CPC außerordentlich viel an Material, das hektographiert wurde und in der Regel 24 Stunden nach Schluß der Debatten bei den Zeitungen eintraf.

Das Material wird wie folgt charakterisiert:

„Täglich einen oder zwei politische Leitartikel, bearbeitete Auszüge aus den Berliner Blättern, eine Wochenschau, aktuelle Feuilletons, und vor allen Dingen einen Parlamentsbericht über alle Verhandlungen des Deutschen Reichstages und des Preußischen Landtages unter Beruicksichtigung der Zentrums-Redner. Ferner Berichte über alle wichtigen Kongresse, namentlich die Katholikenversammlungen, sodann über Gerichtsverhandlungen und dergleichen. Dazu vertrauliche Informationen nur für die Gesellschafter."17

Als ihre publizistische Hauptaufgabe betrachtete die CPC die Förderung der Kommunikation zwischen den Zentrums-Fraktionen und der Presse; das natürlich nicht ohne Blick auf Leser und Wähler.

Auf diese Weise trug die CPC jahrzehntelang dazu bei, die Politik der ZentrumsPartei der Presse und dem Leser transparent zu machen, das Verständnis der Wähler gegenüber der Partei und die oft gerühmte Geschlossenheit des Zentrums $\mathrm{zu}$ erhalten und $\mathrm{zu}$ verstärken. Diese Leistung ist um so bemerkenswerter als wie schon festgestellt - die Unabhängigkeitsbestrebungen der Zentrumspresse und die oft unverständliche Informationspolitik der Partei die Arbeit der CPC erschwerten. 
Der Vorwurf der mangelnden Informationsbereitschaft auf Seiten der Partei läßt sich beispielsweise dadurch erhärten, daß weder die Fraktionen der Partei noch das erst 1918 gegründete Reichsgeneralsekretariat über einen Pressesprecher verfügten und keine Kontakt- bzw. Mitteilungsinstitution bestand..$^{18}$

Niemals, und das ist - zumindest bis 1920 - entscheidend für die Arbeit der CPC, war die Korrespondenz parteioffiziell Teil des Kommunikationssystems zwischen Wählern, Presse und Partei, nicht zuletzt deshalb, weil sie eben immer eine Institution der Presse selbst war, als solche gegründet und geführt; als solche auch dann noch handelnd und behandelt, als die Partei sie 1920 offizialisierte.

\section{Neukonstruktion und Ende der CPC nach dem 1. Weltkrieg}

Die organisatorischen Vorschläge, die in der Eingabe der CPC und des AV 1917

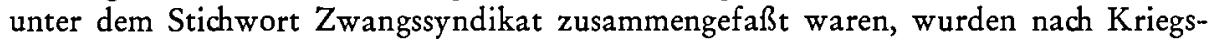
ende und Konsolidierung der Verhältnisse in Partei und Staat wiederaufgenommen und in einer Denkschrift vom 24. 3. 1919 präsentiert. Das neue demokratische Fundament der jungen Republik lieferte dafür eine Reihe von Argumenten, die aus der Erkenntnis der Bedeutung der Presse und ihrer öffentlichen Aufgabe im demokratischen Staat entstanden. So forderte die Denkschrift unter Hinweis auf die bisherigen Erschwernisse den Aufbau einer offiziellen Parteikorrespondenz, für die man der Partei die CPC als Mantel zur Verfügung stellte. ${ }^{19}$

Auf dem Parteitag des Rheinischen Zentrums reagierte die Partei erstmals mit ungewöhnlicher Einsicht und Eindringlichkeit. Eine bemerkenswerte Grundsatzerklärung erfolgte auf dem 1. Reichsparteitag am 21. 1. 1920 in Düsseldorf. Teile aus dieser Erklärung wurden als Entschließung angenommen.20

Darin kam der völlig veränderte Standpunkt der Partei zu ihrer Presse zum Ausdruck. Vorgesehen waren: Ständige Konsultationen und Kontakte zwischen allen Parteigremien und allen Presseinstitutionen; Einrichtung eines Koordinierungsausschusses in Sachen Presse; Aufbau einer parteioffiziellen Parlamentskorrespondenz, die für alle Zentrums-Zeitungen obligatorisch sein sollte; Absicherung des finanziellen Risikos der Korrespondenz durch die Partei.

Die Entschließung endete:

„Um die rechte Verbindung zwischen den Zentrumsfraktionen, insbesondere der Reichstagsfraktion, und der Presse herzustellen, werden durch Vereinbarung zwischen dem Reichsparteivorstand und dem Vorstand der Reichstagsfraktion auf der einen Seite und der politischen Organisation der Zentrumspresse auf der anderen Seite besondere Einrichtungen getroffen, deren $Z_{\text {weck }}$ die Herausgabe einer offiziellen zentrumspolitischen Korrespondenz (CPC) ist. “21

Auf Drängen der CPC und des AV wurde auf diese Entschließung hin die alte CPC-GmbH sofort aufgelöst und am 2. 2. 1920 in Düsseldorf die neue Korrespondenz, CPC oder jetzt ZPK geheißen, gegründet. Die neun Mitglieder im Aufsichtsrat der neuen CPC-GmbH - drei vom Vorstand der Zentrums-Partei, drei vom $\mathrm{AV}$, drei Verleger von Zentrums-Zeitungen - vertraten je ein Drittel des Gesellschaftskapitals.

Die Weiterführung der redaktionellen Arbeit, im Programm der alten CPC entsprechend, besorgte deren langjähriger Chefredakteur, Franz Fortmann.

So gestrafft und endlich auch in die Arme der Partei geschlossen, begann die CPC/ZPK aufs neue ihre Arbeit. Mit der Abspaltung der Bayerischen Volkspartei22 
und dem Auflagenschwund der Zeitungen in den frühen 20er Jahren begannen jedoch wieder - nun rein finanziell bedingte - Schwierigkeiten des Unternehmens, so daß vom 2. Reichsparteitag der Zentrumspartei (Januar 1922) berichtet wird: „... man beschloß einen Ausbau der CPC. Aber aus Mangel an Mitteln ist er nicht mehr möglich gewesen, ja, es ist sogar ein Rückgang zu verzeichnen. ${ }^{{ }^{23} 3}$

So erwies sich auch die neue Gesellschaft als finanziell zu schwach, um in dieser wirtschaftlichen Situation bestehen zu können. Infolge der verstärkten Depression waren bald die meisten Blätter nicht mehr in der Lage, ihre Abonnements aufrechtzuerhalten und zogen auch ihre Gesellschaftsanteile zurück.

Die Ereignisse müssen sich hier verschränkt und ïberstürzt haben, jedenfalls ist kein offizieller Auflösungsbeschluß belegt, keine eindeutige Darstellung der Situation gegeben, so daß sich vorerst nur feststellen läßt: die CPC/ZPK-GmbH löste sich mit Wirkung vom 1. 7. 1922 auf.

Damit brach die einheitliche parlamentarische Berichterstattung für alle - oder zumindest einen Großteil - der Zentrumsblätter zusammen.

\section{Anmerkungen:}

1. Im Folgenden hauptsächlich nach:

Kisky, Wilhelm: Der Augustinusverein zur Pflege der katholischen Presse Deutschlands von 1878-1928, Festschrift zum 50. Jubiläum, Düsseldorf 1928. Originale der CPC konnten nur in wenigen Ausgaben in der Stadtbibliothek Mönchengladbach beigebracht werden. Die CS-Redaktion bittet interessierte Leser um Hinweise: Wo befinden sich noch größere Bestände von CPC-Materialien, - evtl. in Privatbesitz?

2. Heitjan, F. E.: Die ,Saar-Zeitung und die Entwidklung des politischen Katholizismus an der Saar von 1872-1888, Phil. Diss. Köln, Saarlouis 1931, S. 75 f.

3. Kisky, a.a.O., S. 64

4. Bachem, Karl: Vorgeschichte, Geschichte und Politik der deutschen Zentrumspartei, 8 Bde., Köln 1927-1931, Bd. III, S. 152 f.

5. Kisky, a.a.O., S. 239 f.

6. Bachem, K.: Vorgeschichte ..., Bd. III, S. $152 \mathrm{ff}$.

7. Vgl. Kisky, a.a.O., S. 80.

8. Vgl. Kisky, a.a.O., S. $167 \mathrm{ff}$.

9. Otto, Wilhelm: Rede auf dem Katholikentag Köln 1903, vgl. Verhandlungen der 50. Generalversammlung der Katholiken Deutschlands in Köln vom 23. bis 27. 8. 1903, Köln 1903, S. 397.

10. Morsey, Rudolf: Die Deutsche Zentrums-Partei 1917-1923, Düsseldorf 1966, S. 605 f.

11. Kisky, a.a.O., S. 134 ff.

Kisky meint hier Pressedienste wie die "Klausner'sche Korrespondenz" in Berlin, von der schon vor 1874 die meisten katholischen Zeitungen ihre Berichte bezogen.

12. Kisky, a.a.O., S. 129 ff.

13. Lensing, Lambert: Rede zum 25jährigen Bestehen des Augustinus-Vereins, Katholikentag Köln 1903, a.a.O. (vgl. Anm. 9), S. 258.

14. 1871 hatte es in der Rheinprovinz und in Westfalen 44 Zentrums-Zeitungen mit ca. 100 Tsd. Beziehern gegeben, 1912 waren es 180 Blätter mit 1,5 Mio Beziehern. Nadh Bachem, K., Vorgeschichte..., Bd. III, S. 157 f.

15. Kisky, a.a.O., S. 171.

16. Gemeint ist hier der Preußische Landtag.

17. Kisky, a.a.O., S. 129 f.

18. Morsey, a.a.O., S. $605 \mathrm{f}$.

19. Kisky, a.a.O., S. $171 \mathrm{ff}$.

20. Morsey, a.a.O., S. 605.

21. Löffler, K.: Geschichte der katholischen Presse Deutschlands, M.Gladbach 1924, S. 87 ff.

22. Im April 1920 spaltete sich der Bayerische Landesverband der Deutschen Zentrumspartei zur Bayerischen Volkspartei ab und gründete im Juni 1920 eine eigene parlamentarische Korrespondenz.

23. Löffler, a.a.O., S. 90. 